\title{
PIEZOELECTRICIDAD Y FERROELECTRICIDAD EN BIOMATERIALES
}

\section{PiezoeleCtRiCity AND FERROELECTRICITY IN BIOMATERIALS}

\author{
Alejandro Heredia ${ }^{1,2}$, Igor Bdikin² y María Colín-García ${ }^{1,3}$
}

\author{
${ }^{1}$ Instituto de Ciencias Nucleares Universidad Nacional Autónoma de México, Circuito Exterior C.U. 04510 México, D.F., \\ México. \\ ${ }^{2}$ Department of Ceramics and Glass Engineering, CICECO, University of Aveiro, 3810-193 Aveiro, Portugal. \\ ${ }^{3}$ Instituto de Geología, Universidad Nacional Autónoma de México, Circuito Exterior C.U. 04510 México, D.F., México. \\ Autor para correspondencia: aheredia@nucleares.unam.mx
}

Manuscrito recibido el 28 de septiembre de 2011. Aceptado, tras revisión el 10 de octubre de 2011.

\section{Resumen}

En este artículo se presenta una revisión de los conceptos de piezoelectricidad y ferroelectricidad, así como sus aplicaciones en materiales complejos e híbridos orgánico-inorgánicos mediante el uso de las técnicas de microscopía de fuerza atómica (AFM) y de fuerza de piezorespuesta (PFM). Aunque este tipo de estudios son escasos en las áreas biológicas, se sugiere el uso de estas técnicas para analizar las propiedades de piezorespuesta en péptidos debido a su relevancia en las reacciones prebióticaspero y en el diseño de máquinas nanomoleculares.

Palabras claves: Piezoelectricidad, ferroelectricidad, biomoléculas, AFM, PFM.

\begin{abstract}
The present article is a review of the concepts of ferroelectric and piezoelectric materials, as well as the application in complex compounds such as inorganic-organic hybrids using atomic force microscopy (AFM) and piezoresponse force microscopy (PFM). Even there are no many reports of these kinds of studies on the biological area, we suggest that the AFM and PFM techniques could reveal the piezoelectric properties of peptides. Those properties are relevant in prebiotic reactions and potentially applicable on the design of nanomolecular engines.
\end{abstract}

Keywords: Piezoelectricity, ferroelectricity, biomolecules, AFM, PFM

Forma sugerida de citar: Heredia, A., I. Bdikin y M. Colín-García. 2011. Piezoelectricidad y ferroelectricidad en biomateriales. La Granja. Vol. 14(2): 3-12. ISSN: 1390-3799. 


\section{Introducción}

Los materiales ferroeléctricos constituyen una subclase dentro de los materiales piezoeléctricos, es decir, son materiales que experimentan una deformación mecánica cuando se les aplica un voltaje, o que por el contrario se cargan eléctricamente cuando son deformados. Los materiales ferroeléctricos exhiben una gran gama de propiedades funcionales incluidas: alta polarización eléctrica, piezoelectricidad fuerte, actividad óptica no lineal, piroelectricidad sobresaliente, y comportamiento no lineal dieléctrico. Estas propiedades son indispensables para su aplicación en numerosos dispositivos electrónicos como sensores, detectores infrarrojos, filtros de microondas y, recientemente, memorias no volátiles, por nombrar solo algunas. Debido a la combinación única de propiedades, los investigadores e ingenieros se han centrado en la visualización y crecimiento de los dominios ferroeléctricos (zonas de polarización) a diferentes escalas (Jesse et al., 2007).

Los recientes avances en la síntesis y la fabricación de materiales ferroeléctricos a micro y nanoescala dan vida a nuevos fenómenos físicos, por lo que los dispositivos deben ser estudiados y comprendidos también a estas escalas. Cuando las dimensiones estructurales se hacen más pequeñas, los materiales ferroeléctricos presentan un pronunciado efecto dependiente del tamaño, el cual se manifiesta en una desviación de las propiedades de las estructuras de baja dimensionalidad respecto de sus análogos a gran escala (Ahn et al., 2004). En este sentido, los ferroeléctricos son similares a los materiales magnéticos, pues la energía superficial tiene que ser considerada para volúmenes pequeños y de largo alcance debido a la interacción de los dipolos, la cual origina el comportamiento ferroeléctrico.

A raíz de los problemas de miniaturización (i.e., nanotecnología), se ha requerido el uso de nuevas técnicas para la evaluación de las propiedades ferroeléctricas y piezoeléctricas con alta resolución. Problemas fundamentales actuales como por ejemplo el efecto ferroeléctrico y piezoeléctrico, la relación entre micro y macrorespuestas con la estabilidad, o el crecimiento y la degradación del dominio ferroeléctrico son de vanguardia y sus soluciones pueden llegar a representar la respuesta a muchas incógnitas en distintas aplicaciones como el cómputo de alto rendimiento.

Más allá de las aplicaciones nanoescalares, la funcionalidad de las películas ferroeléctricas, de las cerámicas, e incluso de los monocristales, depende de la comprensión de la nucleación del centro ferroeléctrico y la importancia de los defectos, como las grietas presentes en la estructura (Alexe y Gruverman, 2004). Esto ocurre porque los defectos pueden favorecer el desarrollo de núcleos ferroeléctricos y, por lo tanto, determinar la piezorespuesta o la distribución de los mismos núcleos. Para entender los mecanismos fundamentales que generan los núcleos ferroeléctricos y su posterior funcionalidad, es necesario estudiar las estructuras a escala nanométrica. Por ello, el rápido desarrollo de la microscopía de fuerza atómica (AFM; por sus siglas en ingles) (Binnig et al., 1982) y una modificación de ésta, la microscopía de fuerza de piezorespuesta (PFM) se ha traducido en un gran adelanto en el análisis nanoescalar. En la sección siguiente se detallan los principios físicos básicos de estas microscopias.

\section{Principios básicos de la mi- croscopía de fuerza de piezo- respuesta $(\mathrm{PFM})$}

Las propiedades piezoeléctricas y ferroeléctricas a nivel nanoescalar pueden ser exitosamente evaluadas por PFM (Brukman y Bonnell, 2008). El efecto que se evalúa es la asociación entre la polarización y el desplazamiento mecánico de la muestra como se indica en la Figura 1.

Como se puede apreciar, existe un acoplamiento entre la expansión-contracción y campo eléctrico en la muestra. La aplicación de un campo eléctrico muy puntual (i.e., localizado) sobre el material puede ser inducido por el microscopio AFM. La exploración de los desplazamientos resultantes puede llevarse a cabo mediante mediciones topográficas, de fase y de fuerzas con precisión micro y nanométrica como se indica en la Figura 2.

Debido a la gran resolución vertical, la microscopía AFM representa una plataforma ideal para el análisis local de la piezoelectricidad de los materiales. Esta característica, junto con la alta concentración del campo eléctrico, puede ser medida por el sensado (i.e., contacto) de la punta del microscopio con la topografía de la muestra en estudio. No obstante, el microscopio AFMPFM también puede detectar el acoplamiento electromecánico local. Como ocurre en la microscopía AFM, el mecanismo básico de formación de imágenes en el PFM está fundamentado como complementario a la fuerza de interacción punta-superficie. 


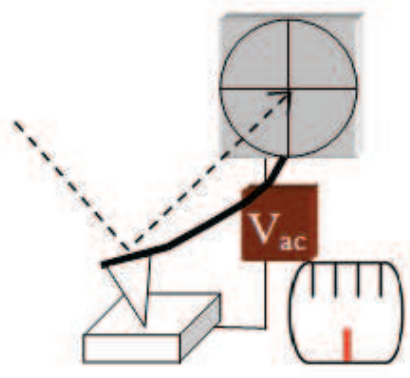

Voltímetro

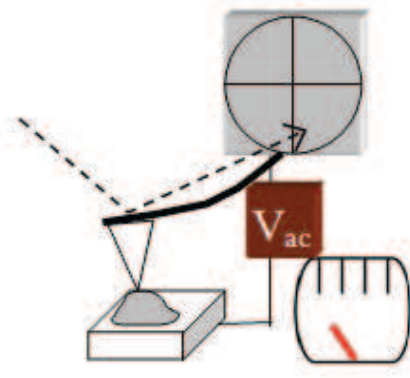

Voltímetro

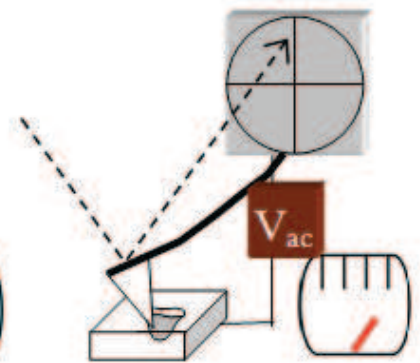

Voltímetro

Figura 1. Aplicando un voltaje de corriente alterna, la punta del microscopio AFM puede producir una disminución o aumento del área topográfica medida, la cual a su vez es sensada por la punta del AFM produciendo una imagen de polarización piezoeléctrica independiente a la imagen de topografía. El voltaje (representado aqui por la aguja en el monitor del voltímetro) polariza (i.e., expande o contrae) a la superficie de la muestra dependiendo del signo de la corriente.

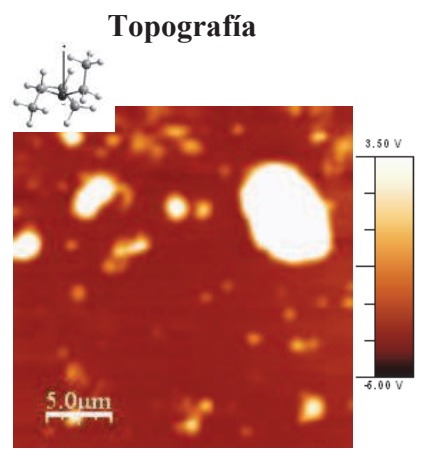

Polarización fuera de plano

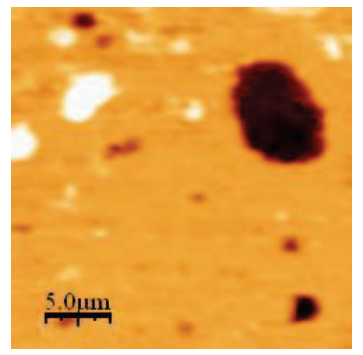

Polarización en el plano

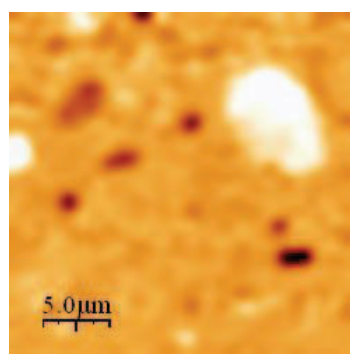

Figura 2. Imagen de trietanolamina (dentro de la imagen de topografía) depositada en una superficie conductora de oro. Se muestran los resultados de la topografía, las polarizaciones fuera del plano y en el plano. Las imágenes de las polarizaciones indican que al aplicar un voltaje DC sobre la superficie, existe un desplazamiento de la muestra, demostrándose así las propiedades piezoeléctricas del compuesto.

\subsection{Origen y funcionamiento de la mi- croscopía de fuerza piezoeléctrica, PFM}

Tras la invención del microscopio de efecto túnel (STM, por sus siglas en inglés) (Binnig et al., 1982), y del microscopio AFM, los primeros experimentos de piezoelectricidad de los materiales relacionados con su deformación inducida por la aplicación de un voltaje DC a través de la punta de los microscopios fueron realizados en 1991. En estos primeros experimentos se estudió la piezorespuesta de los materiales mediante el uso de la microscopía de barrido acústico y STM (Birk et al., 1991; Guthner et al., 1991). Posteriormente, aparecieron los primeros trabajos de AFM relacionados con las mediciones y modificaciones de piezoelectricidad y de visualización de dominios ferroeléctricos (Franke et al., 1994). Dichos experimentos arrojaron rápidamente toda una serie de resultados pioneros de gran importancia, de los cuales los más representativos se reportaron por Takata (Takata et al., 1994), Franke (Franke et al., 1994) y Gruverman (Gruverman et al., 1996). Los estudios de Gruverman son particularmente importantes 
porque se pudo demostrar que mediante la polarización y las imágenes obtenidas siempre era posible observar el cambio en los dominios ferroeléctricos comunes, acuñándose desde entonces el término piezorespuesta, y desarrollándose activamente la microscopia de fuerza de piezorespuesta (PFM, por sus siglas en inglés).

El funcionamiento de un microscopio PFM se basa en la activación de la superficie de la muestra mediante la aplicación de un voltaje desde la punta del microscopio. Existen dos tipos de voltaje, los cuales dependiendo de la polaridad; por un lado está la polarización que no cambia de signo (DC) y por otro lado la polaridad que sí lo hace (AC). Esto es importante pues es la base de la detección de las propiedades eléctricas de los materiales; si se presenta piezoelectricidad "sensada" por el voltaje AC, o si presenta ferroelectricidad "sensada" por el voltaje DC).

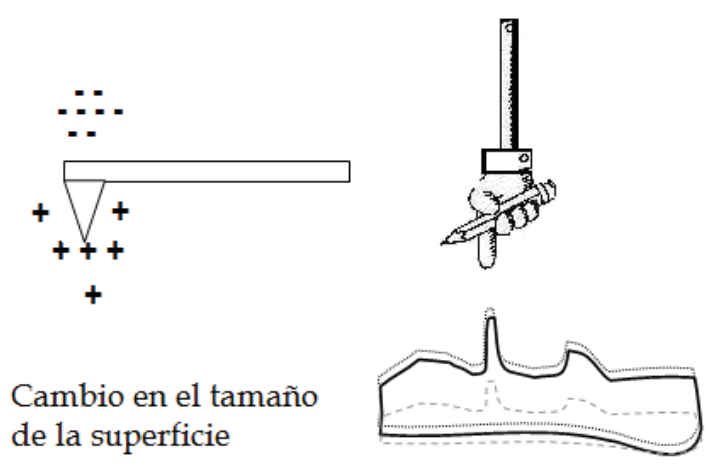

Figura 3. Gráfico esquemático del comportamiento de la punta sensora piezoeléctrica

\subsection{Piezorespuesta}

Cuando se aplica un voltaje AC en particular, se obtiene una piezorespuesta específica. La punta del microscopio AFM es desplazada sobre la superficie "sensando" diferentes dominios con distintas polaridades, y como respuesta se obtiene imágenes topográficas de las regiones de la superficie de la muestra que han cambiado de dimensión. Cuando la muestra varía de tamaño, debido a la piezoelectricidad inducida, la punta del AFM modifica su posición (se eleva o baja; se desplaza hacia la izquierda o la derecha), y así para controlar este cambio de posición se usa un amplificador sensible a fase (lock-in amplifier) de manera que la oscilación de la punta tenga un valor constante. A continuación se explica el movimiento básico de detección.

Sea el voltaje aplicado desde el equipo a la punta: $V_{t i p}=V_{D C}+V_{A C} \cos (\omega t)$ donde, $V_{A C}$ s la polarización alterna que sensa los cambios en la superficie de la muestra, y $\omega$ es la frecuencia de funcionamiento (driving frequency).

Dado que la muestra bajo estudio se está expandiendo y contrayendo, por fenómenos de piezoelectricidad inducidos por la aplicación de un voltaje directo, entonces la punta del AFM también se mueve a fin de mantener la resolución vertical. Este movimiento vertical de la punta es monitoreado por amplificador sensible al cambio de la fase del movimiento de la punta (lock-in amplifier), lográndose relacionar el comportamiento de la punta a una oscilación del tipo: $A=A_{0}+A_{1 \omega} \cos (\omega t+\varphi)$ donde $A_{0}$ es el cambio en el tamaño de la superficie, $\varphi$ es la diferencia de fase entre el voltaje de funcionamiento, sensible a cambios en la superficie (driving voltaje), $V_{A C}$ el voltaje que induce la deformación en la superficie $\left(A_{1 \omega}\right)$. Finalmente, la deformación de la superficie puede expresarse como

$$
A_{1 \omega}=d_{33}^{e f f} V_{A C}+\frac{\partial C}{\partial z}\left(V_{D C}-V_{S}\right) V_{A C}
$$

donde el primer término es la respuesta piezoeléctrica descrita por el coeficiente effectivo piezoeléctrico $d_{33}^{\text {eff }}$; el segundo término es la deformación electrostática causada por tensiones no maxwellianas. $V_{S}$ está relacionado con la energía potencial superficial y $C$ es la capacitancia total del sistema punta-muestra.

Con la información de PFM, obtenida directamente del instrumento, se obtienen varios tipos de imágenes. Por un lado, se obtiene la amplitud, la cual es generada por la información de la magnitud del acoplamiento local electromecánico obtenida; y la fase, la cual está relacionada con la orientación de la polarización. Estos dos tipos de imágenes no solo son de gran calidad por su gran resolución vertical, sino que son de mucha utilidad la cual puede ayudar a determinar la mitad de la extension de un dominio piezoeléctrico, $D P=A_{1 \omega} \cos (\varphi)$, que es la parte más comunmente usada para la caracterización $\left(\operatorname{con} \varphi \approx 0^{\circ}, 180^{\circ}\right)$. El límite de la resolución está dado por el área de contacto punta-muestra (lo que en las las cajas comerciales de las puntas de AFM viene especificado como radio de la punta) más las interacciones adicionales que varían con este contacto, por ejemplo las interacciones electrostáticas y las fuerzas de adhesión. 


\section{El microscopio de fuerza de piezorespuesta}

\subsection{El contraste}

El mecanismo de generación de contraste y la subsecuente detección de dominios (i.e., regiones) ferroeléctricos con el PFM, se basa en el hecho de que los materiales ferroeléctricos también son necesariamente piezoeléctricos. La punta realiza tres tipos de movimiento: (1) desviación vertical, que es una consecuencia de la fuerza vertical en la deformación de la superficie (coeficiente $d_{33}^{\text {eff }}$ y polarización fuera del plano en Figura 2); (2) torsión (causada por el coeficiente piezoeléctrico $\left.d_{33}^{\text {eff }}\right)$ y, (3) encorvadura (buckling; causado por la interacción de la punta con la superficie, cuando la fuerza actúa a lo largo del eje del cantiléver). Si la polarización y el campo eléctrico aplicado son paralelos, entonces la deformación es positiva (expansión) y así, la señal de piezorespuesta estará en fase con $V_{A C}$. Por el contrario, si el campo eléctrico aplicado es antiparalelo a la polarización, la muestra piezoeléctrica se contraerá llevando la punta del AFM hacia abajo, es decir, el campo eléctrico y la señal piezoeléctrica tienen un cambio de fase en $180^{\circ}$.

La dirección de la polarización para el caso de la polarización en el plano se determina mediante el coeficiente $d_{33}^{\text {eff }}$. Para este caso en particular, el campo eléctrico aplicado causa una deformación en el dominio piezoeléctrico, el cual es "sensado" por las fuerzas de arrastre de la punta (encorvadura). A esta información se le denomina movimiento "en el plano" u horizontal, la cual provoca el movimiento de las moléculas debido a la interacción de sus momentos dipolares en consecuencia con la ferroelectricidad producida. Como es de suponerse, se requiere de conocimientos básicos de la operación de un microcoscopio de fuerza, para una buena interpretación de los datos de PFM. De lo contrario, se generarían interpretaciones erróneas del comportamiento de las superficies de los materiales.

De la misma manera, se requiere un conocimiento básico de la química y la física asociada a los materiales bajo estudio; especialmente el caso de los materiales compuestos por elementos orgánicos-inorgánicos, así como los compuestos cristal-polímeros, los complejos producto de autoensamblaje, y los materiales de origen biológico que por su alta complejidad estructural y comportamiento electromecánico son difíciles de interpretar.
La complejidad de los materiales de origen natural y la complejidad de la señal del instrumento hacen muy difícil la adquisición de imágenes fuera y en el plano. No obstante, estas imágenes son obtenidas a partir de la señal eléctrica de barrido de las muestras; en ocasiones estas señales o los mismos momentos dipolares están mezclados, haciendo más difícil el estudio de las muestras. Actualmente se está trabajando en nuevos dispositivos que adquieran simultáneamente las señales y que éstas se puedan separar adecuadamente.

\subsection{Evaluación de la ferroelectricidad por PFM}

La importancia del cambio del orden molecular reside en su capacidad de aplicación en las tecnologías computacionales; en los sistemas biológicos constituye quizá una de las formas de acumulación de información. Como en las memorias ferroeléctricas en estado sólido, los polímeros y otros sólidos de gran peso molecular también pueden responder a la aplicación de voltajes DC con el objetivo de cambiar el ordenamiento molecular intrínseco. En el proceso de formación de un núcleo de moléculas alineadas mediante campos eléctricos (i.e., polarización) el inicio está marcado por una cantidad crítica de moléculas que cambian su orden espacial. En este proceso es necesario controlar tanto el tiempo de uso del campo eléctrico como la intensidad del campo, para posteriormente estudiar, modelar e interpretar el comportamiento del crecimiento de nucleo ferroeléctrico. Los experimentos en los que se cambia el tiempo o la intensidad de exposición al campo eléctrico proveen la información del tipo de material ferroeléctrico de que se está estudiando. En materiales crecidos epitaxialmente, así como en cristales, se ha observado que el tamaño del dominio ferroeléctrico aumenta linealmente con el voltaje aplicado de forma logarítmica con el tiempo. Para estos casos existen dos modelos que pueden explicar dicho comportamiento, de los cuales los modelos de crecimiento en el plano toman la idea de activación térmica con una dependencia de la velocidad del crecimiento de la pared del dominio de forma exponencial al campo eléctrico: $\nu=\exp (-1 / B)$. Este modelo describe limitadamente los resultados experimentales para ciertos tamaños de dominio ferroeléctrico ya que también se ha observado que al aumentar los dominios también se generan desviaciones en otras dimensiones del material. Un modelo más general sugiere que la pared del dominio ferroeléctrico se desplaza gradualmente coordinado por un potencial puntual. La velocidad de creci- 
miento del dominio se expresa como: $\nu=\exp (-1 / E) \mu$, con un exponente dinámico $\mu$ que refleja el tamaño del dominio y la posibilidad de desorden estructural.

\section{Los sistemas biológicos y la respuesta piezoeléctrica}

La piezoelectricidad que se deriva de una estructura cristalina no centrosimétrica es una propiedad intrínseca de la mayoría de los péptidos y de muchos polisacáridos. La piezoelectricidad es por tanto un comportamiento muy común en biología, especialmente en los biominerales de origen animal y vegetal. Intentar comprender la interacción entre los campos eléctricos, por ejemplo del sistema nervioso o el potencial eléctrico de la célula y las modificaciones de estos potenciales debido a estímulos mecánicos es muy complejo, pero es una de las principales motivaciones en el estudio de la piezoelectricidad y de la ferroelectricidad en los sistemas biológicos. Este interés no es meramente teórico, pues el hecho de que las biomoléculas y sólidos de origen biológico sean electromecánicamente activos puede ser utilizado en nuevas tecnologías como el desarrollo de sensores a nivel nanométrico. Por ejemplo, se puede llevar a cabo un anclaje covalente de anticuerpos y a la vez evaluar su posterior cambio electromecánico al interactuar con el objetivo (e.g., virus, antígenos, etcétera). Otra aplicación es el desarrollo de computadoras biológicas sensibles a los cambios de campos eléctricos y a la capacidad de mantener la posición de las moléculas en el espacio (i.e., chip biológicos). Se ha observado recientemente (Rosenman et al., 2011), que péptidos pequeños de dos aminoácidos autoensamblados en tubos tienen muy alta piezoelectricidad (comparable al $\mathrm{LiNbO}_{3}$, uno de los materiales más utilizados como transductor inorgánico). Estos nanotubos peptídicos poseen excelentes propiedades electromecánicas. Otro caso en que la ferroelectricidad o la piezoelectricidad puede ser de suma importancia en el área biológica, es el caso de los lípidos y péptidos, los cuales sensibles a cambios de voltaje, como se ha observado en las neuronas y en los músculos. Debido a la alta resolución vertical de los microscopios PFM, estas biomoléculas podrían ser estudiadas con grandes aumentos, determinando si sufren degradación total o parcial, o bien si se deforman o arreglan en el espacio, dependiendo del voltaje administrado. Finalmente, de manera general se puede afirmar que muchas biomoléculas son una magnífica fuente de potenciales nuevas tecnologías, como para la obtención de energía a partir de nuestros propios movimientos al caminar, o para el diseño de celdas solares a nanoescala.

\subsection{Biomoléculas con piezoelectricidad y ferroelectricidad}

Los fosfolípidos son moléculas formadoras de bicapas y de micelas que al autoensamblarse tienen una gran similitud con las membranas celulares (Figura 4) pues poseen dominios moleculares, "rafts" (ver Figura 5) (Eigen, 1971). Estas moléculas juegan un papel activo en la estabilidad de la estructura, pues tienen una región polar con un momento dipolar asociado, lo cual les provee de la capacidad de acoplamiento a fin de poder generar propiedades electromecánicas. Ese podría ser el caso del acoplamiento entre lípidos y proteínas en las neuronas, lo cual genera la ferroelectricidad para condensar en estas moléculas la información estructural a partir de las características fisicoquímicas de cada una (Leuchtag, 2007). Con esta información se puede sugerir que el acoplamiento de fosfolípidos, también puede servir como transductor para obtener información desde el ambiente. El mecanismo posible es la existencia de una cierta periodicidad en el arreglo de las capas lipídicas, además de que el contacto entre las moléculas puede acoplar a los momentos dipolares. Por ejemplo, estudios con L- $\alpha$-fosfatidilcolina autoensamblada demuestran que se puede obtener un potencial al cambiar la inclinación de las moléculas. Este efecto ocurre debido principalmente al autoensamblaje entre las moléculas quirales de los fosfolípidos, en la misma forma como lo harían en un cristal líquido. No obstante, el cambio en la inclinación de esas moléculas es el origen del potencial al cambiar la simetría. El valor encontrado de polarización al inclinar $5^{\circ}$ es ca. $300 \mathrm{nC} / \mathrm{cm}^{2}$ y se propone que este efecto piezoeléctrico puede acoplarse a unidades funcionales biológicas y así poder convertir los estímulos mecánicos en eléctricos o viceversa (Jákli et al., 2008). Además, se ha señalado que este mecanismo puede estar relacionado con la funcionalidad de los biominerales magnéticos para convertir señales magnéticas en fisiológicas.

Otro caso interesante es el de las alas de las mariposas que están compuestas de alfa quitina y mediante PFM-AFM (Binetti et al., 2009; Kalinin et al., 2006) se determinó que poseen piezoelectricidad. Las alas de las mariposas no tienen estructuras cristalinas inorgánicas como en el caso de los huesos o los dientes, donde también se halla este fenómeno en que la colágena tipo I parece ser la responsable. 


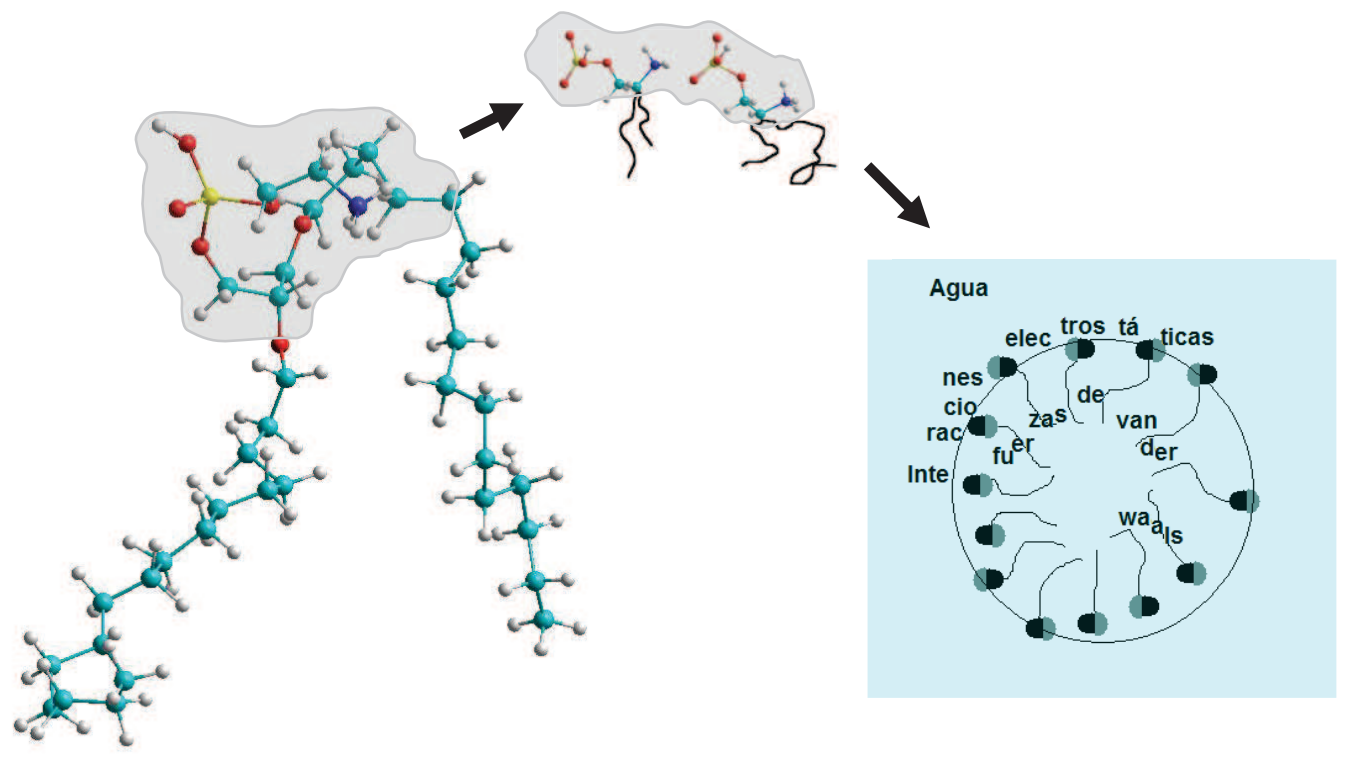

Figura 4. Estructura de un lípido (izquierda) mostrando en la porción sombreada la región con momento dipolar generado (en el modelo molecular fue calculado en 3.9 Debye) por la región amino y la de fosfato. Se muestra también el autoensamblamiento de dos fosfolípidos en agua (en medio) formando una micela (derecha) producto de la interacción de las regiones hidrofóbicas en las moléculas mediante fuerzas de van der Waals.

\subsection{Aplicaciones de la PMF en los es- tudios sobre el origen de la vida}

La polaridad es una propiedad de muchas de las moléculas biológicas. En algunos casos, ésta se da porque dentro de la misma molécula existen cargas positivas y negativas, que generan una diferencia en la densidad de cargas. Dentro de este grupo de compuestos se encuentran los aminoácidos, los cuales siempre presentan dos regiones cargadas: un amino residual $(\mathrm{N}+)$ y otro carboxilo (C-); además, muchos aminoácidos también presentan cadenas laterales ionizables. Estas características hacen que cuando un péptido se autoensamble al mismo tiempo presente una carga parcial, la cual varía en función de las propiedades fisicoquímicas ambientales. Esto, por supuesto determinará la forma en que un péptido o una proteína interacciona con el medio (Veis, 2003). En el caso particular de los aminoácidos y pequeños péptidos, esta carga natural permite la interacción con distintas superficies, entre ellas los minerales. Por ejemplo, se sabe que las arcillas de naturaleza 2:1, como la montmorilonita, cuentan con cargas parciales positivas expuestas principalmente en las orillas de las estructuras cristalinas, mientras que las cargas negativas se presentan en los canales. Estas cargas distintas pueden llegar a favorecer e incluso imposibilitar la interacción con otras moléculas. Por ejemplo, dentro del canal interlaminar, el cual está cargado negativamente, las moléculas positivas son fácilmente adsorbidas (ver Figura 5a).

Los aminoácidos poseen cargas en el estado sólido (ver Figura 6). Más particularmente, los aminoácidos polares con carga neta positiva (e.g., histidina, arginina, lisina) pueden alojarse en estos canales. Por el contrario, los aminoácidos cargados negativamente (e.g. ácidos aspártico, glutámico) podrían estar interaccionando con las orillas de estos minerales. La interacción de los aminoácidos con las arcillas ha sido estudiada desde hace varias décadas (Greenland et al., 1962) observándose que a $\mathrm{pH}$ ácidos y concentraciones altas se adsorben exitosamente debido al intercambio catiónico y a la generación de puentes de hidrógeno. En general, las características de los aminoácidos como el punto isoeléctrico, el peso molecular y el tamaño favorecen estas interacciones (Ponnamperuma et al., 1982).

La interacción de minerales con la materia orgánica es un fenómeno común en la naturaleza. De este tipo es la relación que existe entre minerales como la apatita y las proteínas (colágena) en el hueso de los vertebrados, puede modificar la estructura molecular de la colágena 

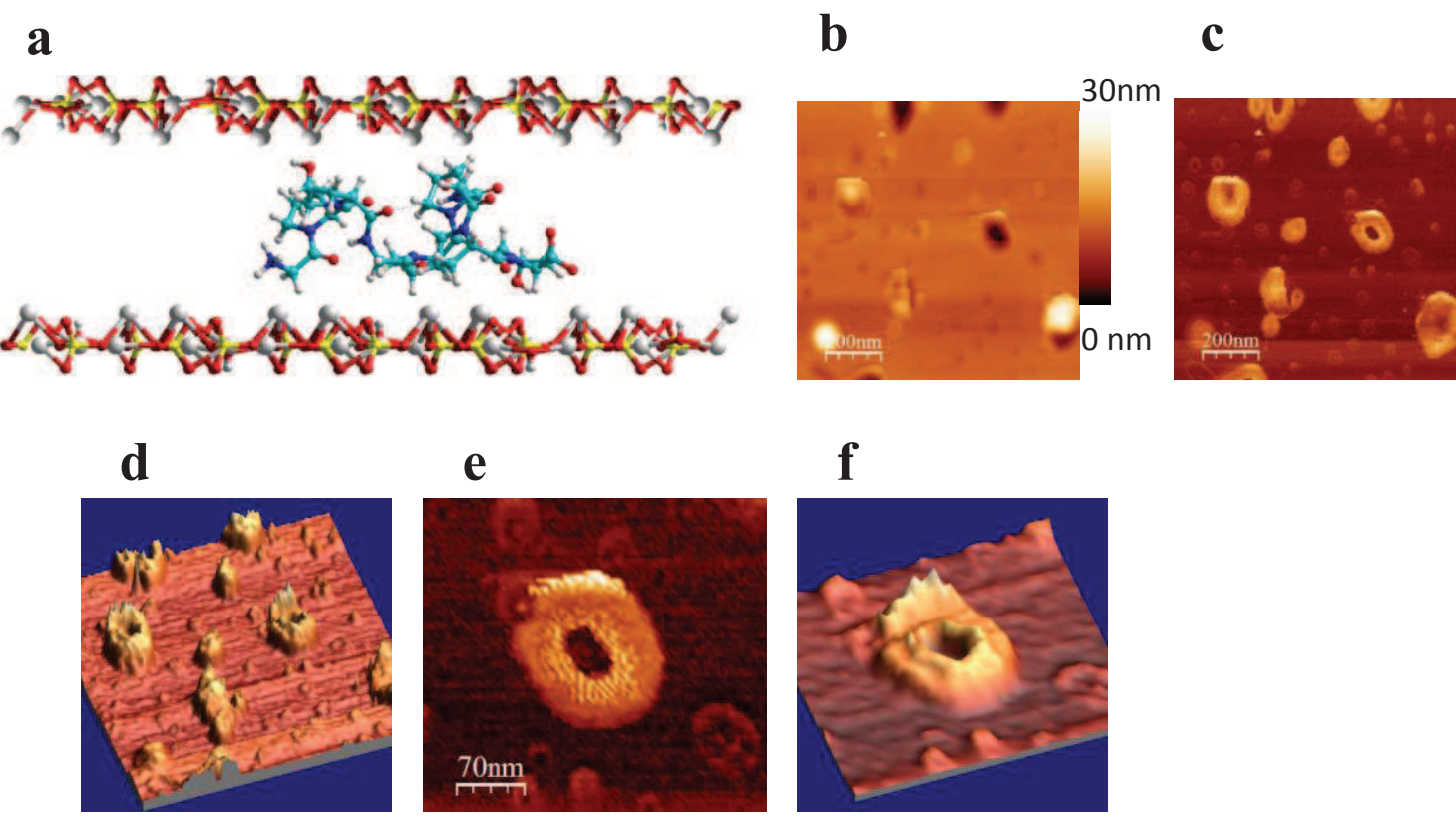

Figura 5. El autoensamblamiento en superficies inorgánicas (a) coadyuva a ordenar las moléculas dependiendo de la distribución de cargas en la superficie.

generando piezoelectricidad (Minary-Jolandan y Yu, 2009); además, se ha postulado que la asociación entre la materia orgánica del suelo y la fracción mineral pudo haber sido clave en el origen y evolución de la vida en la Tierra. Los autores particularmente estamos interesados en este último fenómeno así como en el aprovechamiento del potencial tecnológico de la microscopía de fuerza piezoeléctrica con el objeto de estudiar los posibles mecanismos del autoensamblaje molecular mediante la piezoelectricidad. Por ejemplo, es muy conocido el hecho (desde el punto de vista prebiótico) que la interacción mineral-compuesto orgánico debió haber sido importante en la organización tridimensional de monómeros de la glicina (Figura 5) (Goldman et al., 2010), y cuyas fases cristalinas presentan piezoelectricidad (Lemanov, 2000).

A partir de materias primas muy sencillas (e.g., aminoácidos) y con la ayuda de la piezoelectricidad, se pudo haber promovido la síntesis de numerosos compuestos complejos en los primeros instantes de la Tierra primitiva. Subsecuentemente, estos compuestos pudieron haber reaccionado para formar a las moléculas prebióticas que culminaron con el surgimiento del primer organismo. Sin embargo, los compuestos sintetizados al mismo tiempo eran degradados y su concentración pudo haber sido críticamente baja.

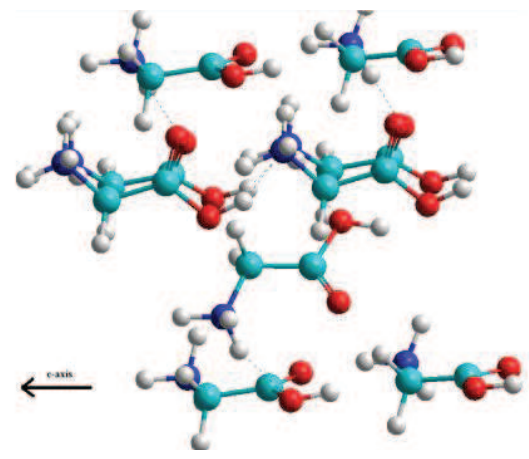

Figura 6. La gama glicina presenta polarización intrínseca en su estructura cristalina, es decir, las cargas positivas están orientadas en una dirección en todo el espacio y las negativas igualmente, haciendo posible generar propiedades electromecánicas.

De manera independiente Goldschmidth (Goldschmidt, 1952) y Bernal (Bernal, 1949) sugirieron que las arcillas podrían haber jugado un rol importante en la síntesis prebiótica. Por una parte, Goldschmidth sugirió que debido a la muy baja concentración de las moléculas orgánicas en la Tierra, la adsorción de éstas 
en las superficies minerales podría haber sido clave para la generación de agregados más ordenados. Aunque muchos han sido los experimentos para simular estas condiciones (Eigen, 1971), aún estamos lejos de entender los procesos que facilitan o limitan las interacciones entre las moléculas orgánicas y las superficies inorgánicas. Por ello, creemos que el desarrollo y la aplicación de técnicas como la microscopía PFM pueden ser adecuadas para entender a detalle los fenómenos electromecánicos involucrados en estas interacciones. La información derivada de estas fuentes también podría abrir un nuevo campo sobre el entendimiento del origen de la vida en la Tierra.

\section{Conclusiones}

La evolución de la familia de la microscopia de sonda y fuerza ofrece una gran gama de posibilidades para la obtención de información referente a las propiedades de los materiales. En particular, la microscopia PFM (empleada inicialmente en el estudio de los dominios ferroeléctricos de materiales inorgánicos) ha demostrado también ser útil en el estudio de materiales más complejos (i.e., híbridos orgánicos-inorgánicos). En forma particular, es interesante el estudio por PFM de los materiales de origen biológico debido al claro y versátil acoplamiento de las cargas e iones del medio y al posible orden molecular biológico generado. No obstante, este tipo de estudios puede tener aplicaciones en otros campos de la ciencia como por ejemplo, en el estudio del origen de la vida. Actualmente, los estudios de la evolución química todavía no han considerado las propiedades ferro y piezoeléctricas de las moléculas orgánicas implicadas en la evolución (e.g., aminoácidos, péptidos). En este trabajo se ha revisado la utilidad de la microscopia PFM en el estudio de las propiedades de biomoléculas implicadas en el origen de la vida. La microscopía de respuesta piezoeléctrica traerá nuevas respuestas a estos retos donde la colaboración entre físicos, químicos y biólogos resulta de vital importancia.

\section{Referencias}

Ahn, C. H., K. M. Rabe y J. M. Triscone. 2004. Ferroelectricity at the nanoscale: Local polarization in oxide thin films and heterostructures. Science, 303: 488-491.

Alexe, M. y A. Gruverman. 2004. Nanoscale charac- terisation of ferroelectric materials: Scanning probe microscopy approach. Springer.

Bernal, J. D. 1949. The physical basis of life. proceedings of the physical society. Section B, 62: 597.

Binetti, V. R., J. D. Schiffman, O. D. Leaffer, J. E. Spanier y C. L. Schauer. 2009. The natural transparency and piezoelectric response of the greta oto butterfly wing. integrative biology. 1: 324329 .

Binnig, G., H. Rohrer, C. Gerber y E. Weibel. 1982. Tunneling through a controllable vacuum gap. Applied Physics Letters, 40: 178-180.

Birk, H., J. Glatz-Reichenbach, L. Jie, E. Schreck y K. Dransfeld. 1991. The local piezoelectric activity of thin polymer films observed by scanning tunneling microscopy. Fifth international conference on scanning tunneling microscopy/spectroscopy, 2 ed. AVS, Boston, Massachusetts (USA): 1162-1165.

Brukman, M. J. y D. A. Bonnell. 2008. Probing physical properties at the nanoscale. Physics Today, 61: $36-42$.

Eigen, M. 1971. Selforganization of matter and the evolution of biological macromolecules. Naturwissenschaften, 58: 465-523.

Franke, K., J. Besold, W. Haessler y C. Seegebarth. 1994. Modification and detection of domains on ferroelectric pzt films by scanning force microscopy. Surface Science, 302: L283-L288.

Goldman, N., E. J. Reed, L. E. Fried, I. F. William Kuo y A. Maiti. 2010. Synthesis of glycinecontaining complexes in impacts of comets on early earth. Nat Chem, 2: 949-954.

Goldschmidt, V. M. 1952. Geochemical aspects of the origin of complex organic molecules on the earth, as precusors to organic life. New Biology, 12: $97-105$.

Greenland, D. J., R. H. Laby y J. P. Quirk. 1962. Adsorption of glycine and its di-, tri-, and tetrapeptides by montmorillonite. Transactions of the Faraday Society, 58: 829-841.

Gruverman, A., O. Auciello y H. Tokumoto. 1996. Nanoscale investigation of fatigue effects in 
pb(zr,ti)o[sub 3] films. Applied Physics Letters, 69: 3191-3193.

Guthner, P., J. Glatz-Reichenbach y K. Dransfeld. 1991. Investigation of local piezoelectric properties of thin copolymer films. Journal of Applied Physics, 69: 7895-7897.

Jesse, S., S. V. Kalinin, R. Proksch, A. P. Baddorf y B. J. Rodriguez. 2007. The band excitation method in scanning probe microscopy for rapid mapping of energy dissipation on the nanoscale. Nanotechnology, 18: 435503.

Jákli, A., J. Harden, C. Notz y C. Bailey. 2008. Piezoelectricity of phospholipids: a possible mechanism for mechanoreception and magnetoreception in biology. Liquid Crystals, 35: $395-$ 400.

Kalinin, S. V., B. J. Rodriguez y J. Shin. 2006. Bioelectromechanical imaging by scanning probe microscopy: Galvani's experiment at the nanoscale. Ultramicroscopy, 106: 334-340.

Lemanov, V. V. 2000. Piezoelectric and pyroelectric properties of protein amino acids as basic materials of soft state physics. Ferroelectrics, 238: $211-218$.
Leuchtag, H. R. 2007. Voltage-sensitive ion channels: Biophysics of molecular excitability. Springer.

Minary-Jolandan, M. y M.-F. Yu. 2009. Uncovering nanoscale electromechanical heterogeneity in the subfibrillar structure of collagen fibrils responsible for the piezoelectricity of bone. ACS Nano.

Ponnamperuma, C., A. Shimoyama y E. Friebele. 1982. Clay and the origin of life. Origins of Life and Evolution of Biospheres, 12: 9-40.

Rosenman, G., P. Beker y I. Koren. 2011. Bioinspired peptide nanotubes: deposition technology, basic physics and nanotechnology applications. Journal of Peptide Science, 17: 75-87.

Takata, K., K. Kushida, K. Torii y H. Miki. 1994. Strain imaging of lead-zirconate-titanate thin film by tunneling acoustic microscopy. Jpn. J. Appl. Phys., 33: 3193-3196.

Veis, A. 2003. Mineralization in organic matrix frameworks. Reviews in Mineralogy and Geochemistry, 54: 249-289. 